\title{
Lusioersily
}

\section{Origami: a versatile modeling system for visualising chemical structure and exploring molecular function}

Davis, J., Leslie, R., Billington, S., \& Slater, PR. (2010). Origami: a versatile modeling system for visualising chemical structure and exploring molecular function. Chemistry Education Research and Practice, 11(1), $43-47$. https://doi.org/10.1039/C001044H

Link to publication record in Ulster University Research Portal

Published in:

Chemistry Education Research and Practice

Publication Status:

Published (in print/issue): 01/01/2010

DOI:

10.1039/C001044H

Document Version

Publisher's PDF, also known as Version of record

\section{General rights}

Copyright for the publications made accessible via Ulster University's Research Portal is retained by the author(s) and / or other copyright owners and it is a condition of accessing these publications that users recognise and abide by the legal requirements associated with these rights.

\section{Take down policy}

The Research Portal is Ulster University's institutional repository that provides access to Ulster's research outputs. Every effort has been made to ensure that content in the Research Portal does not infringe any person's rights, or applicable UK laws. If you discover content in the Research Portal that you believe breaches copyright or violates any law, please contact pure-support@ulster.ac.uk. 


\title{
Origami: a versatile modeling system for visualising chemical structure and exploring molecular function
}

\author{
James Davis ${ }^{\mathrm{a}}$, Ray Leslie ${ }^{\mathrm{a}}$, Susan Billington ${ }^{\mathrm{b}}$ and Peter R. Slater ${ }^{\mathrm{c}}$ \\ Received 31st March 2009, Accepted 29th September 2009 \\ DOI: $10.1039 / \mathrm{C001044H}$
}

\begin{abstract}
The use of Origami is presented as an accessible and transferable modeling system through which to convey the intricacies of molecular shape and highlight structure-function relationships. The implementation of origami has been found to be a versatile alternative to conventional ball-andstick models, possessing the key advantages of being both inexpensive and readily accessible. The challenge, tangible nature, sense of achievement and hence trophy-like status of the models were found to combine and serve as a potentially invaluable outreach resource in terms of transfer to the home environment, with most (>70\%) participants) wishing to show them to friends and family. A range of models were constructed and their implementation as lecture aids in both secondary and tertiary curricula has been assessed.
\end{abstract}

Keywords: Origami, models, shape, modeling, molecular shape, visualization

\section{Introduction}

Shape has a vital role in the function of biological molecules, and is a critical factor in governing our well being, but the concepts involved in rationalizing why molecules possess a given structure and how it influences their function can be difficult to convey to a non scientific audience. The pilot project outlined herein sought to investigate the development of new modeling concepts based around the art of origami that address this problem through being readily transferable from the classroom to the home. It was our intention that introducing 'take-away' models to school pupils could serve as an ideal conduit through which to stimulate inquiry between pupil and parent, and hopefully from there to the wider community. While molecular modeling kits and computer programs are routinely used within secondary and tertiary education to display 3D molecular architecture (Barnea and Dori, 1996; Dori and Barnea, 1997; Tasker and Dalton, 2006; Aksela and Lundell, 2008), neither approach is readily accessible beyond the educational environment; they often require significant technological expertise, and remain largely abstract to the average person (Aksela and Lundell, 2008).

In developing a 'hands-on' approach to building molecules, it was expected that the pupils would gain a more tangible experience (Jones et al., 2005; Tversky, 2005) that could be retained and shown to friends, parents and other family members. The core rationale of the project was to assess the ability of the model system to convey the significance of geometry, the structural complexity that can be associated with it, and thereby its ability to highlight the significance of shape within a real world context - be it biomedical or

${ }^{a}$ Chemistry, Nottingham Trent University, Nottingham, NG11 8NS, UK E-mail:james.davis@ntu.ac.uk

${ }^{b}$ Chemistry, Dukeries College \& Complex, Whinney Lane, Ollerton, NG22 9TD, UK

${ }^{c}$ Chemistry, University of Birmingham, Edgbaston, Birmingham, B15 2TT, $U K$ industrial. Origami is a modeling system that has traditionally been used as teaching aid in the mathematical sciences (Mitchell, 2005) but it can easily be transformed into a versatile vehicle for explaining concepts central to many biological and chemical systems. In this instance, the intention was to provide activities that could be used to draw parallels between the precision inherent to architecture and function at the molecular scale, but within a challenging framework that fosters an inclusive approach to participation - irrespective of age. The remit was to go beyond the text descriptions and static diagrams and provide a model form that directly engages - more so than simply presenting information (Glenberg and Langston, 1992, Mayer and Gallini, 1990; Mayer et al., 1996). Secondary to the development of the modeling system was the provision of versatile, multi-level resources that places models and associated activities within easy reach of teacher/outreach practitioners, but which could also be used for follow up investigations by the target pupil-parent cohort.

\section{Project methodology}

Origami is traditionally associated with children's leisure activities, and it was envisaged that this principle would serve as a hook for capturing the interest of the pupils. In order to engage the pupils, it was clear that the activities would need to be interactive, fun and challenging, whilst covering the range of abilities across the entire secondary school spectrum. Origami provides a multitude of possible models, and their relevance to molecular architecture can be tailored to specific examples. There are distinct geometrical models (tetrahedral, octahedral and trigonal bipyramidal constructs being among the more obvious) that can be readily mapped to simple molecular shape. Single tetrahedral models can be easily manipulated to show how a three-dimensional shape can be produced from simple building blocks, which can be further developed to generate 'chiral' models. This is highlighted in 

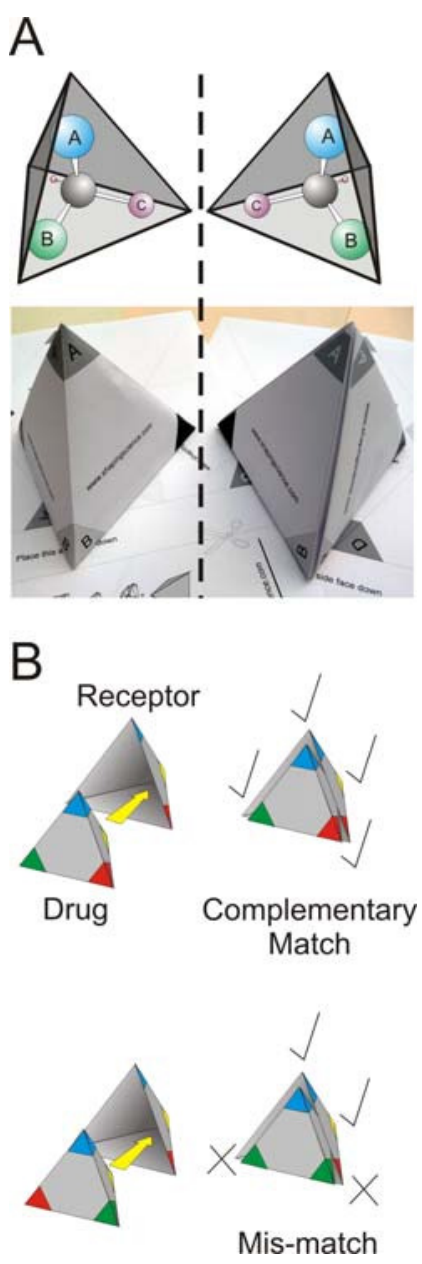

Fig. 1 Introducing chirality using Origami.

Fig. 1A where 'marked' tetrahedra can be used in place of the ball-and-stick moly-mods. Contextual examples can range from basic amino acids to more elaborate case-studies, such as the thalidomide controversy problem. The solid profile of the platonic shapes could, initially, be conceptually difficult for pupils to map to the tetrahedral $\mathrm{sp}^{3}$ carbon. However, the labelling of the apexes and their association with different groups can be used to overcome this issue and strengthen the appreciation of spatial distribution. In addition, more complex origami shapes encompassing skeletal variants can be used to highlight the central atom - whether tetrahedal or octahedral, and can be readily mapped to the conventional ball-and-stick molecular models. Their assembly, however, is substantially more complex, and arguably, beyond beginner level participation, but pre-folded models could be used as corroborative examples by the teacher.

The main message is that a single form can be used at multiple levels - as a fun lecture interlude, but one which can re-focus the class and reinforce the core material (Mayer and Gallini, 1990; Mayer et al., 1996). This could be steadily built upon to introduce the three-dimensional nature of the biochemical world and the significance of chirality in molecular interactions (i.e. enzyme-substrate or drug-receptor

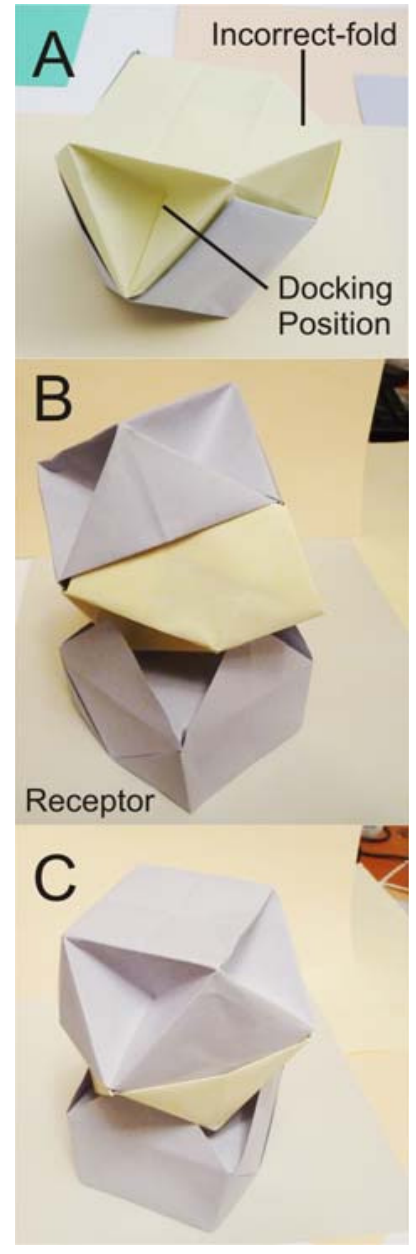

Fig. 2 A) Two-part model with four receptor sites on one face (dark) but only three on the upper face (light). B) Incorrect shape prevents docking. C) All four sites are available - docking with the crown receptor is now possible.

docking), as indicated in Fig. 1B. The tetrahedral shapes discussed previously can be further utilised - in conjunction with an open tetrahedral model - whereby the former fits neatly within the latter and essentially simulates a docking arrangement.

On a separate and much broader level, origami can be used to show how a precise sequence of folds is required to produce a desired, structurally defined object. This mirrors the generation of macromolecular structures that abound in nature, such as polypeptide chains that undergo specific folding processes to produce enzymes, proteins and antibodies. The correct folding sequence is vital for maintaining structure and associated activity, whilst incorrect folding can be responsible for the generation of prions (implicated in 'hole in the brain' disorders such as CJD). This is highlighted in Fig. 2, where an incorrect fold can lead to a marked change in shape that prevents recognition. The versatility of the approach is such that they can be harnessed for explaining the fundamentals at the molecular level, but can also be used to highlight the implications for function at the macroscopic. 
Many of the concepts in these modeling systems have clear parallels with the life sciences, and allow an easy, inexpensive, and creative route through which the pupils can directly visualize structure-function relationships. More importantly, it was expected that the models would provide a more tangible and transferable platform through which the teacher can emphasize the sophistication of the natural world and our current attempts to understand, harness and adapt its creative power.

\section{Pilot study}

The remit of the pilot study was to develop a series of integrated electronic resources consisting of model assembly instructions complete with demonstration video clips, and a range of printable templates (essentially complete origami kits). The teacher would then be able to preview the models, and download the required materials for use within the classroom. A gallery of models would be developed such that teachers would be able to select those examples that are relevant to their own particular teaching programme. Origami, as the core modeling system, was justified on the basis that:

- it provides an enjoyable activity through which a range of pupils of varying ability can participate;

- the models produced can be directly related to molecular shapes that are regularly encountered in biological systems, and increasingly form the basis of new biomolecular engineering research;

- the materials needed to facilitate the activities are inexpensive and are readily available within the schools;

- the approach allows the pupils to physically grasp the models in such a way that the structure-function and complexity issues are reinforced;

- the activities don't require any form of chemicals or specialist equipment, are completely safe, and can be conducted in a variety of locations;

- the resulting models provide a tangible and adaptable resource from which science could be bridged to complementary studies within the school, such as art displays;

- the inevitable transfer of the models from classroom to playground to home offers an opportunity to stimulate wider interest and debate.

Web based delivery of the instructional (model) and associated support materials to schools was selected on the basis that:

- the majority of schools will meet the ICT requirements and thus possess sufficient technology to enable direct access to the resources;

- the main requirement for pursuing the activities relate to the consumption of paper, which is readily available within schools. It also allows individual schools / teachers to be creative in terms of selecting paper styles and the selection of appropriate examples from the web site.

- as an information-based resource, minimal costs are incurred by the receiving institutions;

- the web-based approach allows an effective means through which the underlying concepts (i.e. molecular engineering) can be accessed by a wide audience, and the resources

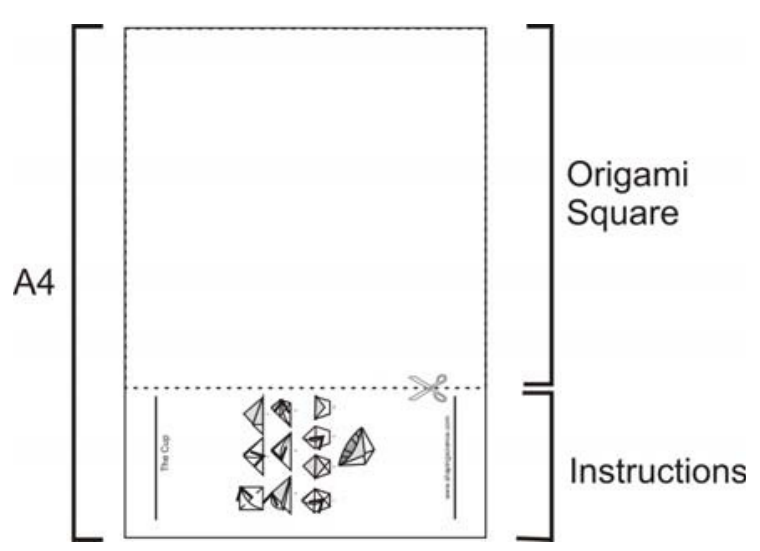

Fig. 3 Single sheet origami kit.

would be available for follow up exploration by pupils and their parents.

\section{Audience}

The main target audience was secondary school pupils (11-17 yrs) and comprised a cohort ideally suited to engaging with the activities. Origami provides a wide spectrum of models and complexities that can encompass the whole of the age range advocated here (and far beyond) and therefore, the challenging aspect of the models can be easily tuned to the audience and resources that are available to the teacher.

\section{Results and discussion}

A website was constructed (www.shapingscience.com) and the basic, step by step instructions for a range of models compiled and made available for screen, print or download. It was initially envisaged that the instructions alone would be sufficient, and that the teachers could easily select the appropriate model and simply print off the required number of copies for their pupils. Initial feedback, however, revealed a number of problems, the main one being the fact that square paper (not A4) is needed to make the models and the provision of such can become a significant burden. The resources are meant to serve as complementary activities for enhancing the learning experience, yet, if the preparation process is too time consuming or difficult in its operation then the uptake will clearly be compromised. The second issue relates to the nature of the instructions - although these were found to be sufficient for the simpler models - initial trials revealed that they were too complicated when dealing with the more elaborate systems, which was due to the number of required steps being too great - especially for the beginner (Marcus et al., 1996).

The paper requirements were quickly solved by the introduction of a complete origami 'kit' on a single A4 sheet. This was done for each model within the gallery, and simply requires the kit to be printed - one sheet per student. The 'square' used for constructing the model is defined on the actual page, with the instructions contained within the section of paper that would normally have been discarded. The basic scheme is shown in Fig. 3, with the square portion easily detachable by the pupils themselves through repeated folding 

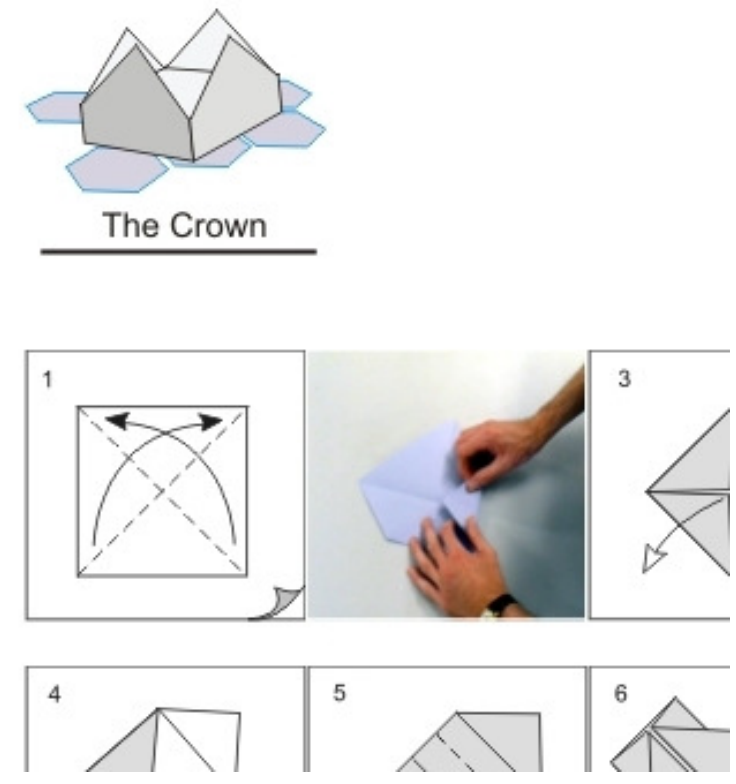

Fig. 4 Interactive model instructions, which flip from schematic to actual picture (www.shapingscience.com)

and final tearing of the pre-printed line. Issues over the clarity of the actual instructions were resolved through the provision of photographic stills for each step and a video clip of the entire assembly process as a means of reducing the cognitive load form one stage to another (Marcus et al., 1996; Novick and Morse, 2000). These can be projected directly from the website by the teacher, whereby each step can be 'flipped' to reveal the corresponding picture (Fig. 4). This can be used to choreograph the assembly process throughout the class, and ensure that all reach completion before moving onto the next stage of the lesson. Alternatively, the video can be played direct, or downloaded and distributed. A key feature is the ability for the files to play on mobile phones. The latter are ubiquitous within the school population and, while their operation within a normal classroom setting will almost certainly be prohibited, their use in this scenario may be of some merit. The phone clips can be viewed individually, paused as required, and thereby allow the pupils to work at their own pace rather than having to constantly replay the demonstration to the entire class. More importantly, they can be taken away and require no internet connection. This is significant in that it may facilitate transfer to the home.

The models come in a variety of shapes and their potential adaptation to a given lecture is only limited by the imagination of the teacher. Preliminary work lead to the development of the 'crown' assembly (Fig. 2), which can help to develop an understanding of receptor architecture and, as was stated previously, this can be used to show the importance of size and shape in models that will interact with the crown, thus mimicking ligand-receptor interaction. This can be introduced as a natural break within normal chalk-and-talk delivery, as the models can be constructed easily within 10 minutes. Some of the other models are more time consuming to construct, particularly those involving modular assembly such as a trigonal bipyramid or multipart cube. These can,
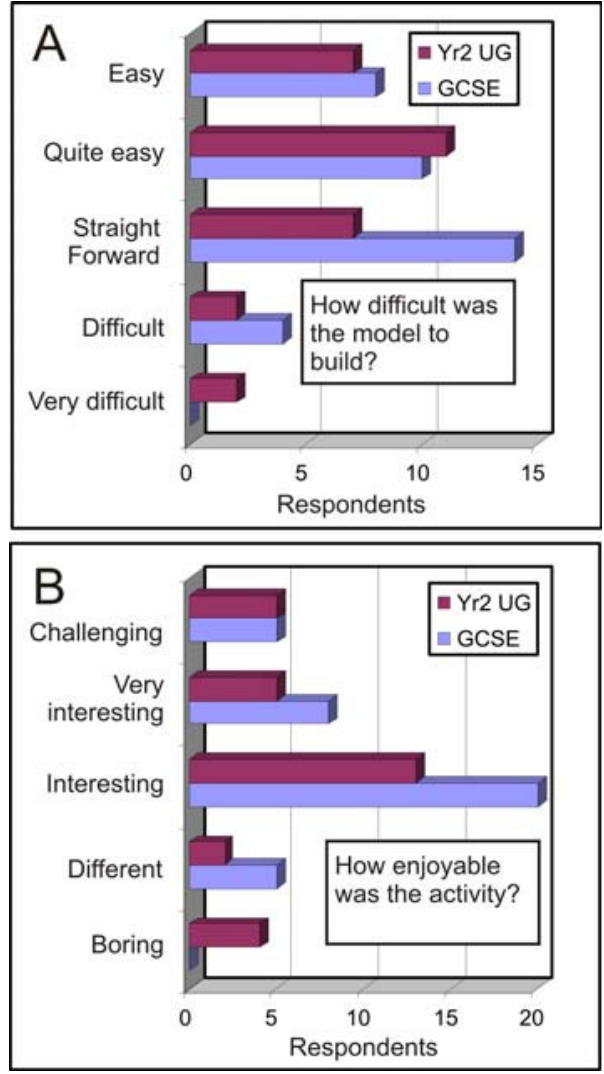

Fig. 5 Responses to queries relating to A) model difficulty and B) activity engagement. GSCE N=38; Second year undergraduates $\mathrm{N}=29$.

however, be integrated into a lesson as a team working session - typically working in groups of 3 or 6 , or individually, where time constraints are less rigorous. They could also be used as a revision / homework aid, again facilitating the transfer of the science to the home. This approach sits in stark contrast to traditional ball-and-stick models, where it would be inconceivable to consider the release of such kits outside the classroom.

Origami is clearly a versatile option for teacher implementation, but the main question is really whether or not the pupils can engage with the models themselves. This was assessed at two levels: with a GSCE revision class (15-16 yrs, $\mathrm{N}=38)$ and with a second year undergraduate class (19-20 yrs, $\mathrm{N}=29$ ) looking at the construction of a modular trigonal bipyramid system. The attitudes of the respective groups to the activity were assessed by questionnaire, and the main findings are summarised in Fig. 5. In both cases, the majority found the activity to be largely interesting, and would appear to support the earlier assumption that the model systems are accessible across a number of age groups. The complexity of the modular system was not found to lead to any significant disenchantment with the activity, but was rather viewed as a challenge. The response to whether or not they would try another puzzle was wholly positive at over $70 \%$, and from those, over $50 \%$ stated they would follow up with accessing the associated internet site.

The average time taken to build this particular model was 25 minutes. Ordinarily this would be too long for a simple 


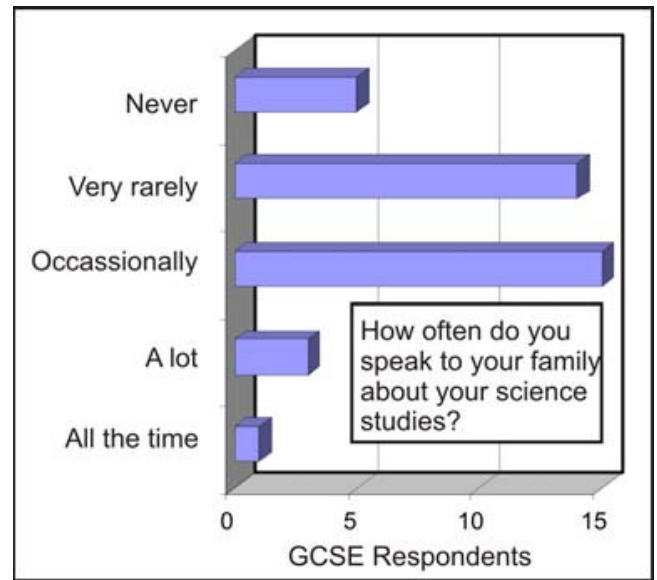

Fig. 6 Transfer of knowledge from school to home.

lecture/lesson interlude, but could be of more use within a tutorial format or as a 'take home and try' activity. The enthusiasm for the latter was also explored, where it was found that over $70 \%$ of the GSCE cohort had stated that they would show the model to either friends or family members (50/50 split). The students were questioned on how often they spoke to their friends/family about their science studies. The results, shown in Fig. 6, are hardly a surprise, but it could be that in many cases there might not be any impetus to bring the subject up. It could be that the completion of the model within the home or simply its transfer to the home, however, could open up the opportunity for further discussion within the family.

\section{Conclusions}

The origami activities trialled thus far have proven to engage the students and provide a tangible outcome that can make its way to the wider community. Therefore, the model system is worth considering as a viable option in a host of teaching platforms. They are not meant to be replacements for the more elaborate ball-and-stick systems nor computer visualizations, but rather complement them. In terms of potential impact there is an important point to note: the sense of achievement in constructing such models at an early age is liable to remain within the memory of the pupils over time. Linking the key concepts of molecular engineering through an almost subliminal route may engender greater interest in the sciences in later life. The activities can be staggered such that some success is guaranteed and thus the possibility of failure generating future negative resonances is avoided. There is a strong possibility that each time they see a paper aeroplane, paper cup, etc. in the future then some reflection on the activities they have engaged in at school or college may occur.

\section{Supplementary material}

The templates for the models shown in Fig. 1 are included in PDF for direct printing and classroom implementation. Templates and associated instructions that allow more flexible user manipulation of the models are available for free download from www.shapingscience.com.

\section{References}

Aksela M. and Lundell J., (2008), Computer based molecular modelling: Finnish school teachers' experiences and views, Chem. Educ. Res. Pract., 9, 301-308.

Barnea N. and Dori Y. J., (1996), Computerised molecular modelling as a tool to improve chemistry teaching, J. Chem. Inf. Comp. Sci., 36, 629-636.

Dori, Y. J. and Barnea N., (1997), In-service chemistry teachers' training: The impact of introducing computer technology on teachers' attitudes and classroom implementation, Int. J. Sci. Educ., 19, 577-592.

Glenberg A. M. and Langston W. E., (1992), Comprehension of illustrated text: pictures help to build mental models, J. Mem. Lang., 31, 129-151.

Jones L. L., Jordan K. D. and Stillings N. A., (2005), Molecular visualisations in chemistry education: the role of multidisciplinary collaboration, Chem. Educ. Res. Pract., 6, 136-149.

Marcus N., Cooper M. and Sweller J., (1996), Understanding instructions, J. Educ. Psychol., 88, 49-93.

Mayer R. E., Bove W., Bryman A., Mars R. and Tapangco L., (1996), When less is more: meaningful learning from visual and verbal summaries of science textbook lessons, J. Educ. Psychol., 88, 64-73.

Mayer R. E. and Gallini J. K., (1990), When is an illustration worth ten thousand words?, J. Educ. Psychol., 82, 715-726.

Mitchell D., (2005), Mathematical origami, Tarquin Publications, St Albans, UK.

Novick L. R. and Morse D. L., (2000), Folding a fish, making a mushroom: the role of diagrams in executing assembly procedures, Mem. Cognition, 28, 1242-1256.

Tasker R. and Dalton R. (2006), Research into practice: visualisation of the molecular world using animations, Chem. Educ. Res. Pract., 7, 141-159.

Tversky B., (2005), Prolegomenon to scientific visualizations, in Visualisation in science education. Models and modelling in science education, J. K. Gilbert (Ed.), Kluwer, Vol 1. pp. 29-42. 